\title{
Structural Analysis of Online Criminal Social Networks
}

\author{
Michael Yip \\ Nigel Shadbolt \\ Craig Webber \\ Web Science Doctoral Training Centre \\ University of Southampton \\ Southampton, SO17 1BJ \\ Email: \{my2e09,nrs\}@ecs.soton.ac.uk, c.webber@soton.ac.uk
}

\begin{abstract}
Over the last decade, a sophisticated underground economy has emerged over the Internet in which cybercriminals collaborate and trade different goods and services. This study takes a unique approach towards understanding the functioning of the underground economy by focusing on the social dynamics between the cybercriminals. Using anonymized private messaging records from four underground forums formerly operating as online black markets, this study aims to examine the structural properties of the networks of personal interactions between the cybercriminals and to turn the findings into actionable intelligence for tackling the problem of profit-driven cybercrime.
\end{abstract}

Keywords-social network analysis; cybercrime; carding; underground economy

\section{INTRODUCTION}

Over the last decade, the nature of cybercrime has shifted from naive vandalism to profit driven and this led to the emergence of a sophisticated underground economy [27]. One of the major sources of profit that is driving this underground economy is carding, a type of identity theft that involves the unauthorized use of credit and debit card account information to fraudulently purchase goods and services [24]. The scope of the term has evolved over the past few years to encompass a broader range of related activities including hacking, phishing and auction frauds. Carders, those who commit carding related crimes, began by using the Internet Relay Chat (IRC) channels $[8,27]$ to trade goods and services with one another. Labor specialization [17] has emerged in this sophisticated "underground economy" with several key roles including carders, cashiers, malware authors, drops, money mules and also, dishonest traders known as the "rippers". These "rippers" represent a "tax" on the goods and services traded in the underground economy [10]. To avoid this "tax", many carders have formed closed knit carding groups in the form of closed membership online forums [33].

In collaboration with the Serious Organised Crime Agency (SOCA) from the U.K., this study takes a unique approach towards understanding the functioning of the underground economy by focusing on the social dynamics between cybercriminals. This could bring about a new set of insights into cybersecurity research because as McIllwain [15] note, "[T]he least common denominator of organized crime is human relationships; specifically human relationships engaged in the process of social networking for the provision of illicit goods and services as well as the protection, regulation and extortion of those engaged in the provision or consumption of these goods and services. This process of social networking occurs as part of a social system of organized crime, a system which explains the remarkable consistency of the process of organizing crime across time and space".

Using anonymized private messaging records from four well known carding forums: Carderplanet, Shadowcrew, Cardersmarket and Darkmarket [9], this study has the unique opportunity to examine the actual social interactions which have taken place in the underground economy. More specifically, by using social network analysis techniques, this paper aims to uncover the underlying network topology of the carding forums, the structural properties of these networks, the behavioral preferences of the cybercriminals and take the first steps towards understanding the network topology of the underground economy as a whole. It is hoped that such insights would allow authorities to better utilize their resources and devise more effective disruption strategies in the future.

\section{MOTIVATION AND RELATED WORKS}

Thomas and Martin [27] were the first to describe the functioning of the underground economy on IRC. Franklin et al performed a detailed quantitative analysis of underground trading on IRC [8]. The underworld of botnets is introduced by Mielke and Chen [16].

The network analysis approach taken in this study is motivated by a number of studies [13, 18, 31, 32], which collectively demonstrate that significant insights into "dark" networks can be found by studying the structure of those networks. This study aims to uncover similar insights in the domains of carding.

Lastly, a recent study analyzed six underground forums including a German carding forum called carders.cc [19]. Their analysis includes both public posts and private messages by using content analysis to examine the impact of user ratings and user behavior. This paper differs from their work by focusing specifically on the topological properties of the private messaging (PM) social network on the carding forums.

\section{RESEARCH DESIGN}

In this study, we have access to the anonymized private messaging (PM) records from four well known carding forums: 
ShadowCrew (SC), Carderplanet (CP), Cardersmarket (CM) and Darkmarket (DM). The forums are deliberately chosen for their chronological order of existence over time which represents the development of carding as an underground business [9]. Their general statistics are shown in table I.

TABLE I. GENERAL STATISTICS OF THE CARDING ForUMS

\begin{tabular}{|c|c|c|c|c|}
\hline & CP & SC & CM & DM \\
\hline Years in operation & $\begin{array}{c}2001- \\
2004\end{array}$ & $\begin{array}{c}2002- \\
2004\end{array}$ & $\begin{array}{c}2005- \\
2007\end{array}$ & $\begin{array}{c}2005- \\
2008\end{array}$ \\
\hline No. of users & 6813 & 4430 & 2759 & 2075 \\
\hline No. of PMs & 28503 & 69126 & 24571 & 15433 \\
\hline
\end{tabular}

\section{A. Carding Forums}

Much like conventional online discussion forums, carding forums are used mainly for trading carding goods and services as those described by Thomas and Martin [27]. However, each forum is further divided into a series of sub-forums which are dedicated to tutorials, discussions and "ripper" lists. Users can start topics, also known as threads, which others can reply to. The forums also offer private messaging functionality and this is of particular interest to this study for two reasons.

Firstly, due to the potential criminal nature of the activities the forum members were engaged in, detailed conversations such as business negotiations were often carried out using private messaging.

Secondly, a private messaging dialogue between two users represents a direct connection between the two and thus can be used to assert the existence of a personal relationship between any pair of the carding forum members. This is a more accurate estimation of the relationships between the forum members than reply graphs which are often constructed using public dialogues [3]. The following section describes the modelling approach used in this study.

\section{B. Private Messaging (PM) Social Networks}

In this study, we model the private messaging (PM) interaction data as a graph where a user is represented by a node and a PM is represented by an edge (undirected) or arc (directed). Two different types of graphs are used for different measurements.

For analyzing degree distribution, assortativity, rich club phenomenon, components, and reciprocity, we model the data as a directed simple graph [29] $\mathrm{G}=\langle\mathrm{V}, \mathrm{E}\rangle$ where:

1. $\mathrm{V}=\left\{\mathrm{v}_{1}, \mathrm{v}_{2} \ldots \mathrm{v}_{\mathrm{x}}\right\}$ is a set of nodes in which each $\mathrm{v}$ represents a member of the forum who have sent or received at least one PM.

2. $\mathrm{E}=\left\{\mathrm{e}_{1}, \mathrm{e}_{2} \ldots \mathrm{e}_{\mathrm{y}}\right\}$ is an ordered set of weighted directed edges (called arcs) and each arc represents a private message sent between two users. The direction of the arc represents the direction of the communication. The weight of an arc represents the number of PMs sent in a particular direction between two nodes.

Thus, the resulting graph is a directed simple graph with no self-loop and no multi-edge between any pair of nodes [29].
For analyzing clustering, diameter, path lengths and cohesive subgroups, we model the data as an undirected graph where the existence of a PM between two nodes is represented by an edge regardless of direction. The weight of an edge is the combined frequency of PMs sent in either direction between two nodes.

\section{RESULTS}

The network properties of the PM social graphs are shown in table II. It can be seen that even with fewer nodes, Shadowcrew hosts the PM social network with most connections, the highest mean degree and highest reciprocity rate.

TABLE II. NETWORK PROPERTIES OF THE PM SOCIAL GRAPHS

\begin{tabular}{|c|c|c|c|c|}
\hline & CP & SC & CM & DM \\
\hline No. of nodes $v$ & 3327 & 2285 & 2149 & 1716 \\
\hline No. of $\operatorname{arcs} a$ & 13429 & 23039 & 11710 & 10149 \\
\hline No. of edges $e$ & 10160 & 14720 & 9433 & 7540 \\
\hline $\mathrm{j}_{\max }$ or $\mathrm{k}_{\max }$ & 123 & 334 & 128 & 196 \\
\hline$<\mathrm{j}>$ or $<\mathrm{k}>$ & 4.04 & 10.08 & 5.45 & 5.91 \\
\hline $\mathrm{x}_{\max }$ & 144 & 353 & 249 & 839 \\
\hline$<\mathrm{x}>$ & 6.11 & 12.88 & 8.78 & 8.79 \\
\hline$i$ & 0.24 & 0.36 & 0.26 & 0.26 \\
\hline$d_{u n}$ & 0.0018 & 0.0056 & 0.0041 & 0.0051 \\
\hline
\end{tabular}

$* \mathrm{j}_{\max }$ is the max in-degree of the network; $\mathrm{k}_{\max }$ is the max outdegree of the network; $\langle\mathrm{j}\rangle$ and $\langle\mathrm{k}\rangle$ is the mean in- and outdegree respectively; $\mathrm{x}_{\max }$ is the max degree of the undirected graph; $\langle\mathrm{x}\rangle$ and $d_{u n}$ is the mean degree and density of the undirected graph; $i$ is the reciprocity of the directed network;

\section{A. Degree Distribution}

First proposed by Barabási and Albert [4], power law degree distributions have since been found to be prevalent in many different types of networks $[6,22]$. Formally speaking, a power law degree distribution is defined by $\mathrm{P}(\mathrm{x}) \sim \mathrm{x}^{-\alpha}$ where the exponent $\alpha$ is free of a characteristic scale, hence systems with such a degree distribution are said to be scale-free [4]. This property is explained by two social processes: growth and linear preferential attachment. To find out whether the carding social networks are scale-free, the Complementary Cumulative Distribution Function (CCDF) of the in-degree and out-degree distribution are plotted, as shown in fig. 1 and 2 respectively. The maximum likelihood estimates of the distribution parameters are given in table III.

To our surprise, it was found that none of the in- and outdegree distributions follow a power law. Rather, they follow a lognormal distribution, an alternative distribution very similar to the power law $[2,6]$. This indicates that non-linear preferential attachment exists in the social networks with a fraction of links established randomly [11,23]. The relative straight tail indicates that preferential attachment exceeds randomness in link distribution [11]. This means that members 

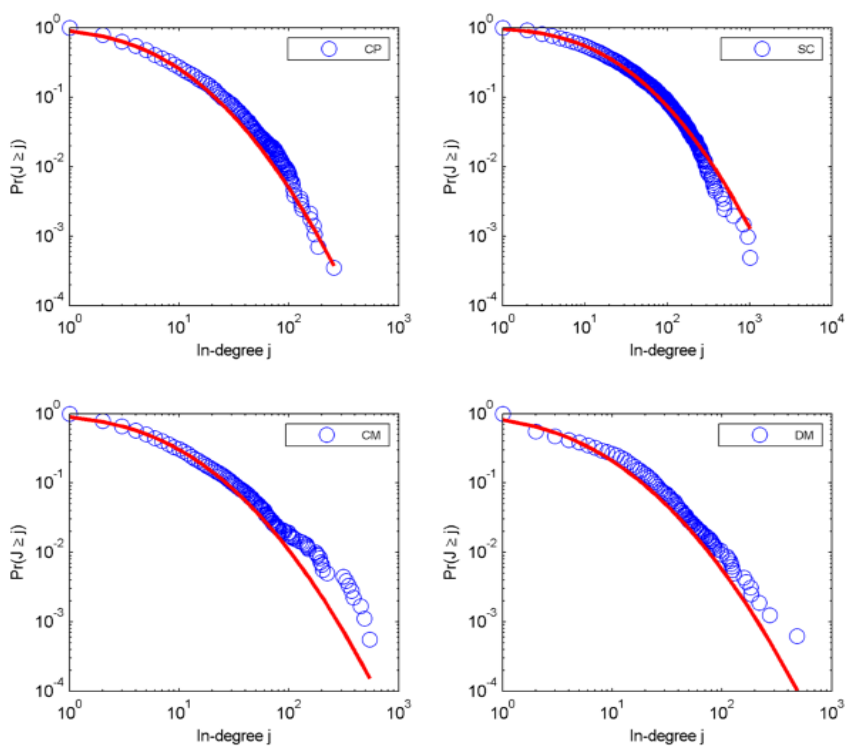

Figure 1. CCDF of in-degree

of the carding forums mostly interact with each other with a specific preference (e.g. reputation of a vendor) but some do interact randomly, perhaps for finding new business opportunities in the wild. This also reflects the mixed nature of carding forums, which operate as online black markets as well as social networking services where members can meet likeminded others.

TABLE III. PARAMETERS OF THE LOGNORMAL DistRIBUTIONS

\begin{tabular}{|c|c|c|c|c|}
\hline & $\mathbf{C P}$ & $\mathbf{S C}$ & $\mathbf{C M}$ & $\mathbf{D M}$ \\
\hline$\mu_{\text {in }}$ & 1.50 & 2.46 & 1.62 & 1.21 \\
\hline$\sigma_{\text {in }}$ & 1.20 & 1.48 & 1.29 & 1.34 \\
\hline$\mu_{\text {out }}$ & 1.68 & 2.42 & 1.85 & 1.83 \\
\hline$\sigma_{\text {out }}$ & 1.22 & 1.46 & 1.37 & 1.29 \\
\hline
\end{tabular}

$*_{\mu}$ is the mean and $\sigma$ is the standard deviation

\section{B. Assortativity}

A network demonstrates assortative mixing if nodes have a preference to associate with others who are similarly well connected [20]. Since the observed network in this case is a communication network via private messaging, the assortativity between the in-degree and out-degree of the senders and recipients are calculated using the following definition as proposed by Newman [21]:

$$
r=\sum_{\mathrm{jk}} \mathrm{jk}\left(\mathrm{e}_{\mathrm{jk}}-\mathrm{q}_{\mathrm{j}}{ }^{\mathrm{in}} \mathrm{q}_{\mathrm{k}}^{\text {out }}\right) / \sigma_{\mathrm{in}} \sigma_{\text {out }}
$$

where $\mathrm{j}$ is the in-degree of the sender, $\mathrm{k}$ is the out-degree of the receiver, q represents the distribution of the relevant excess degree and $\sigma$ represents the relevant standard deviation.

As shown in table IV, $r_{\text {un }}$ represents the correlation between the degree of the sender and the recipient on an undirected graph. All networks are disassortatively mixed, meaning that high degree nodes prefer to connect with low
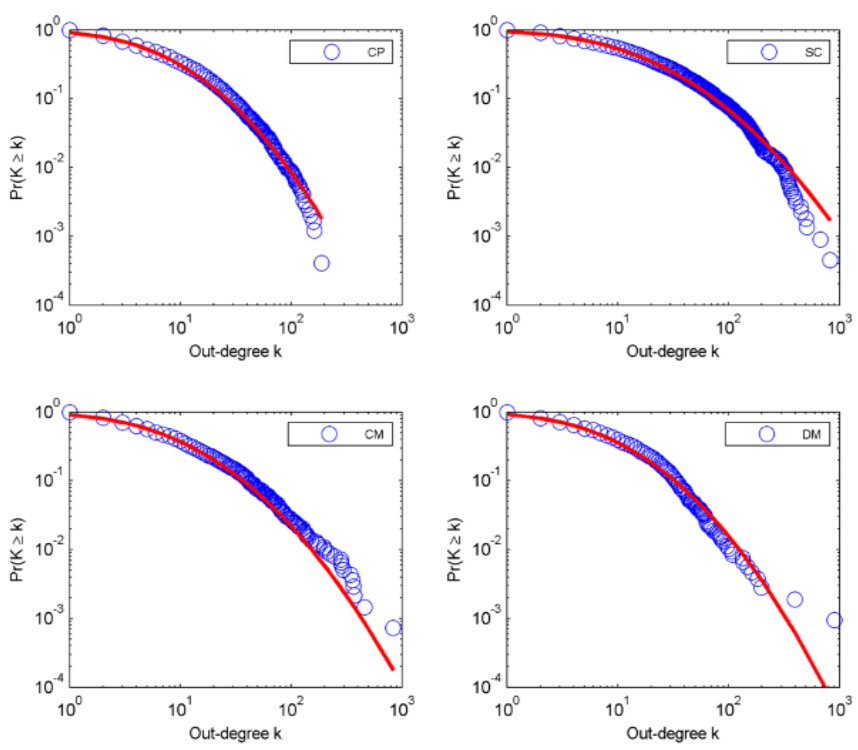

Figure 2. CCDF of out-degree

degree nodes and vice versa. The remaining four assortativity coefficients measure the assortativity of the in-degree and outdegree of senders and recipients on a directed graph, which are all negative.

This suggests that there could be a positive correlation between node-wise in-degree and out-degree. In order to verify this claim, the probability density function (p.d.f.) of the joint in- and out-degree distribution is plotted, as shown in fig. 3. It shows that there is a clear positive correlation between the in-degree and out-degree of each node, meaning that users with low in-degree are also highly likely to have low outdegree and vice-versa.

TABLE IV. AsSORTATIVITY OF NETWORK DEgREE

\begin{tabular}{|c|c|c|c|c|c|}
\hline & $r_{\text {un }}$ & $r_{\text {in-in }}$ & $r_{\text {in-out }}$ & $r_{\text {out-in }}$ & $r_{\text {out-out }}$ \\
\hline $\mathbf{C P}$ & -0.10 & -0.11 & -0.04 & -0.10 & -0.04 \\
\hline $\mathbf{S C}$ & -0.16 & -0.15 & -0.14 & -0.16 & -0.14 \\
\hline $\mathbf{C M}$ & -0.17 & -0.14 & -0.04 & -0.17 & -0.10 \\
\hline $\mathbf{D M}$ & -0.13 & -0.19 & -0.10 & -0.22 & -0.11 \\
\hline
\end{tabular}

Overall, all four carding social networks are disassortative indicating that low-degree nodes tend to connect with highdegree nodes. This suggests that in general, forum members are preferentially attached by connectivity. Indeed, on an asynchronous carding forum, a vendor's in-degree and outdegree is likely to increment proportionally over time because more and more members would see his adverts and contact him privately for negotiations. On the other hand, the buyers who contact the vendors using PM may not be active members of the forum, thus the networks are disassortatively mixed.

\section{Rich-club Phenomenon}

The rich-club phenomenon is a relatively new concept and is defined by Colizza et al [7] as the tendency of high-degree nodes to be very well-connected to each other. In other words, 


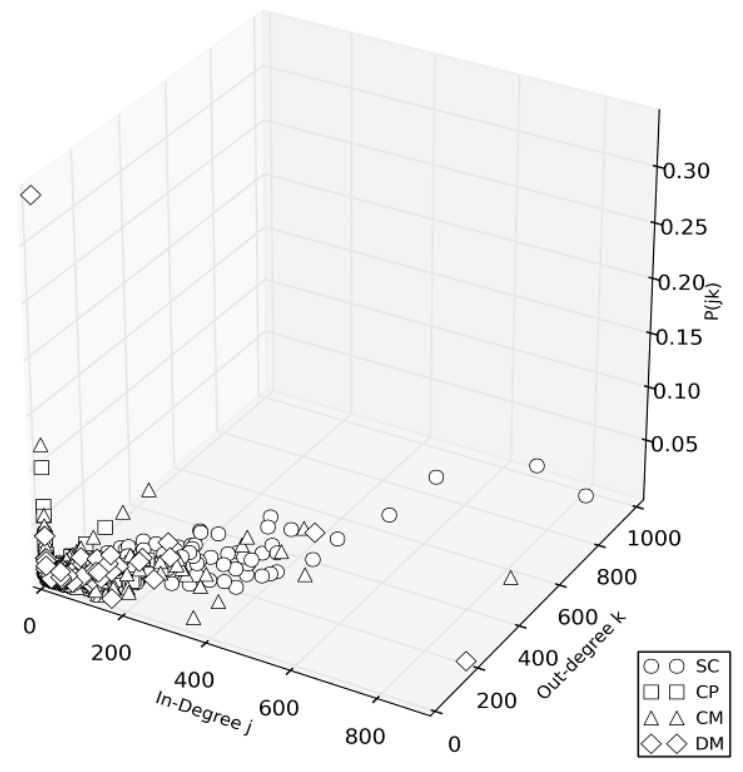

Figure 3. Probability density function (p.d.f.) of joint degree distribution

it measures whether the high-degree nodes are more likely to form well connected subgraphs or "clubs", than low-degree nodes. $\varphi(x)>1$ indicates that there is rich-club behavior whereas $\varphi(x)<1$ indicate that there is an opposing behavior present. It is important to note that rich-club phenomenon can exist in the absence of degree assortativity because it is a measure of the connectivity among high degree nodes, not a measure of the general mixing properties of the network [7]. Here, we use this metric to examine the distribution of disassortativity across the networks.

As shown in fig. 4, all four social networks exhibit similar rich-club behavior where there is an opposing behavior present for nodes with degree of approximately $10^{2}$. This means that these well-connected nodes are not interconnected and this is in direct contrast to the scientific collaboration network [7]. This hints at the presence of a social process that opposes collaboration. Since the carding forums are online black markets, one explanation for this observation is the competition between the vendors.

Furthermore, fig. 4 shows that nodes with the lowest and highest degrees do not exhibit any distinctive behavior. Since new forum members are most likely to have lowest degrees and forum administrators are most likely to have the highest PM degrees, this suggests that members of these two types engage in a balanced mixture of random and preferential interactions.

\section{Transitivity and Small World}

Transitivity measures the likelihood that "the friend of my friend is also my friend" and is commonly referred to as clustering. Two different methods are used to measure the level of clustering in the social graphs: transitivity $T$ [29] and clustering coefficient $C$ [30].

The transitivity $T$ and the clustering coefficient $C$ of the four PM social graphs are as shown in table $V$ which shows
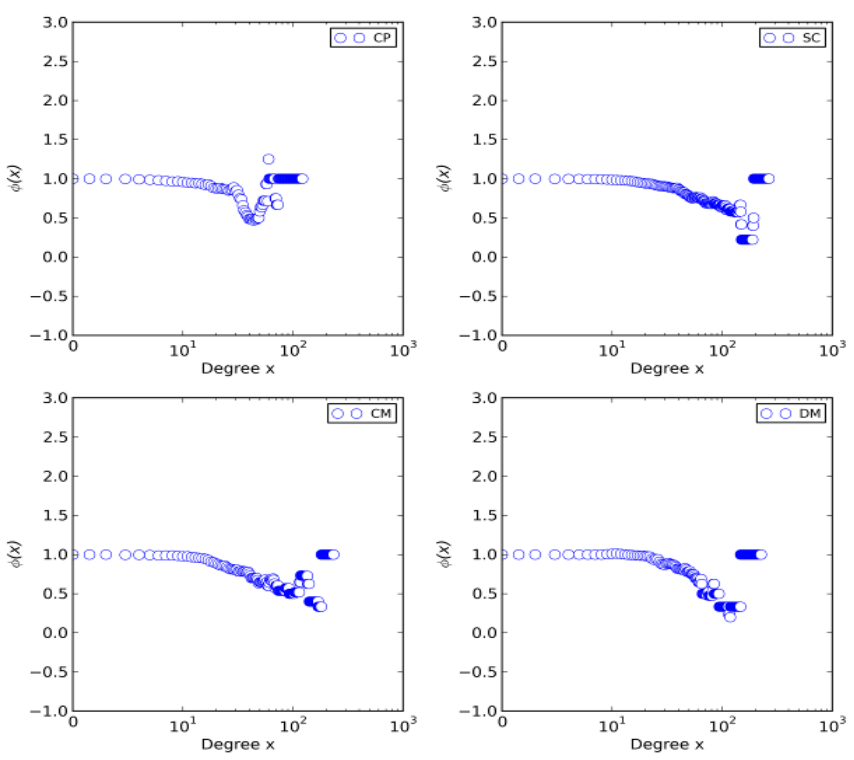

Figure 4. Rich-club phenomenon

that all four PM social networks have a much higher clustering coefficient and transitivity than their random counterparts denoted by $C_{\text {random }}$ and $T_{\text {random }}$. This shows that all four carding forums are highly clustered.

Furthermore, the distribution of the clustering coefficient $\mathrm{C}$ is shown in fig. 5. It is evident from the figure that clustering coefficient is inversely proportional to degree $x$. As Ravasz and Barabási [25] argue, this indicates the existence of a hierarchical architecture in the network.

TABLE V. ClUSTERING COEFFICIENT AND TRANSITIVITY

\begin{tabular}{|c|c|c|c|c|}
\hline & CP & SC & CM & DM \\
\hline$C$ & 0.036 & 0.117 & 0.089 & 0.078 \\
\hline$T$ & 0.034 & 0.066 & 0.048 & 0.032 \\
\hline$C_{\text {random }}$ & 0.0013 & 0.0053 & 0.0039 & 0.0052 \\
\hline$T_{\text {random }}$ & 0.0016 & 0.0052 & 0.0037 & 0.0052 \\
\hline
\end{tabular}

Although the scaling parameter is less than 0.5 in the four networks, which is less than the bounds of 0.75 to 1 suggested by Ravasz and Barabási, this nonetheless suggests that there exists a slight hierarchical architecture. There are two explanations for this: firstly, the carding forums are hierarchically managed with roles such as administrators, moderators, reviewers and reviewed vendors [33]. Secondly, the disassortativity in buyer-seller relationships can also create the hierarchical configuration described in [25].

Lastly, as Watts and Strogatz [30] argue, most real world networks are small worlds as they exhibit two fundamental characteristics: highly clustered like regular graphs and have short path lengths like random graphs. In order to determine whether the four PM social graphs are small worlds, the diameter $D$ and the average shortest path length $p$ are as shown in table VI. It is evident that the four social networks 

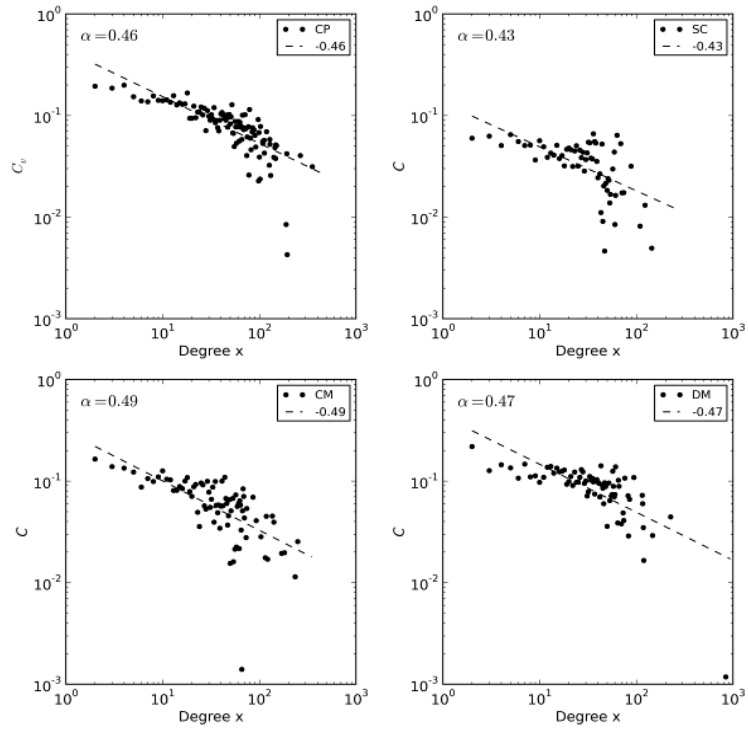

Figure 5. Local clustering coefficient distribution

have approximately identical path lengths as their random counterpart. Thus, all four social networks are small worlds.

TABLE VI. Diameter And Average Shortest Path Length

\begin{tabular}{|c|c|c|c|c|}
\hline & CP & SC & CM & DM \\
\hline$D$ & 9 & 6 & 8 & 7 \\
\hline$D_{\text {random }}$ & 9 & 5 & 7 & 7 \\
\hline$p$ & 4.18 & 3.14 & 3.41 & 2.93 \\
\hline$p_{\text {random }}$ & 4.7 & 3.31 & 3.8 & 3.7 \\
\hline
\end{tabular}

\section{E. Connectivity and Cohesive Subgroups}

The findings on Weakly Connected Components (WCC) and Strongly Connected Components (SCC) are presented in table VII. Like many other social networks [22], we find that each undirected graph consists of a giant WCC with size of over $98 \%$ of the nodes. However, with direction taken into account, we observe a distinctively different pattern. As shown in Table VII, Shadowcrew has by far the largest SCC consisting of over $85 \%$ of all nodes, which is astonishing when compared with other studies on directed networks, such as the World Wide Web [5] and Twitter [12]. This suggests that more dialogues are held using PM on Shadowcrew than the other networks.

TABLE VII. WEAKLY AND STRONGLY CONNECTED COMPONENTS

\begin{tabular}{|c|c|c|c|c|}
\hline & CP & SC & CM & DM \\
\hline No. of WCC & 29 & 3 & 7 & 1 \\
\hline No. of SCC & 1415 & 334 & 1161 & 773 \\
\hline Size of largest WC (\%) & 98.29 & 99.82 & 99.40 & 100 \\
\hline Size of largest SC (\%) & 56.81 & 85.34 & 45.93 & 54.78 \\
\hline
\end{tabular}

Lastly, we examine the connectivity of the graphs using
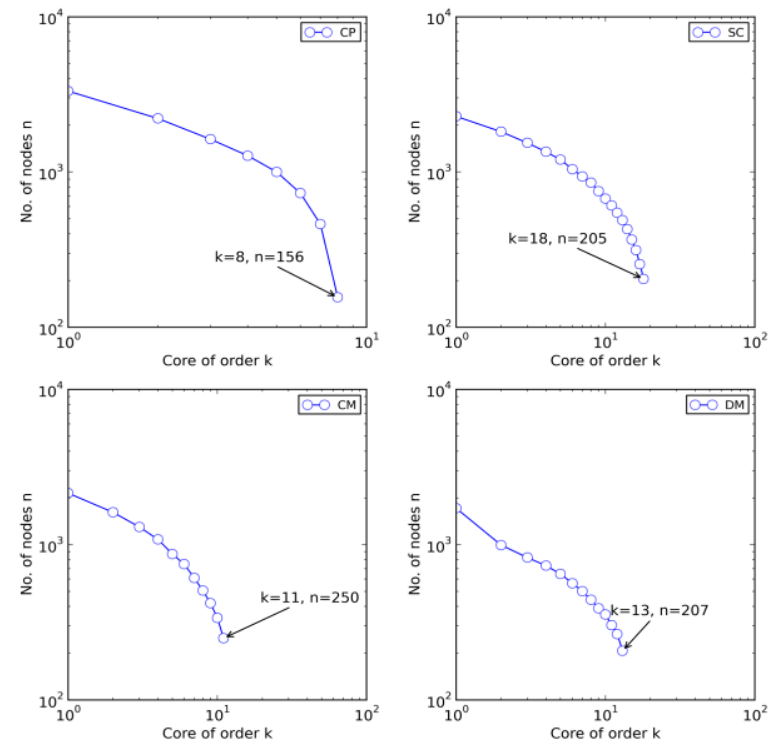

Figure 6. Distribution of $k$-cores

the concept of $k$-core, which is defined as a subgraph in which each node is connected to a minimum of $k$ neighbors [29].

As shown in fig. 6, the rapid decay of $k$-core sizes towards the central core is similar to that of the MSN Messenger social network [14], indicating that each PM network also consists of only a very small dense core. Furthermore, Carderplanet is found to have the smallest range of $k$-cores and as shown in table II, it is the least dense network. On the other hand, Shadowcrew is the densest network with a total of $18 k$-cores. This shows that the number of $k$-cores and the global density of the networks are correlated.

\section{IMPLICATIONS AND DISCUSSION}

In this study, we have found that although there is evidence of preferential attachment and growth, the carding social networks are not scale-free as the degree distributions are lognormal. This indicates the presence of a mixture of random and preferential attachment [11,23] and this has important implications on network disruption. It is widely accepted that scale-free networks are particularly resilient against random node removals [1, 22, 28] but highly vulnerable against targeted attacks because there is only a small fraction of nodes possessing the majority of links. Since the degree distributions in the carding social networks are lognormal, this means that there are less highly connected nodes and more moderately connected nodes [2]. This implies that the networks are more robust against targeted attacks than scale-free networks as well as more resilient against random node removals than random networks [26]. However, targeted attacks remain particularly suitable against carding social networks because they are disassortatively mixed, which means that high- and mediumdegree nodes (e.g. forum administrators and popular members) are well spatially distributed within the network thus the removal of the well connected nodes has a well distributed impact on the entire network [20].

We can also use our findings to speculate on the disruption of carding activities on other channels, such as the IRC. Since 
all carders want to avoid rippers, they would always prefer to trade with reputable or trusted vendors. Hence, reliable vendors are likely to build up large network degree over time. However, without a public reference to the trustworthiness of the vendors as that offered by the forums through rating and reviews, carders on other networks would be forced to interact more randomly in search for business opportunities. Therefore, we believe that the topology of other carding networks are likely to demonstrate stronger lognormal degree distributions, disassortativity and with an absence of rich-club phenomenon. With regards to network disruption, it would be more difficult for law enforcement to target the key individuals on such networks due to the lack of information available. Since many carders operate on forums as well as other networks, we propose that rather than looking to disrupt carding forums, authorities should utilize them for intelligence gathering.

Lastly, the carders have demonstrated a strong need for effective communication by repeatedly choosing to use a hierarchical system such as a forum, despite numerous successful penetrations by law enforcements [9]. Indeed as we have shown, the social networks on these forums exhibit small world characteristics which are ideal conditions for collaboration and information flow [30]. Therefore, it can be said that cybercriminals are willing to trade security for a certain level of efficiency [18]. This suggests that authorities should aim at limiting the number of tools cybercriminals can use to increase their efficiency, such as the use of forums.

\section{ACKNOWLEDGMENT}

We thank the Serious Organised Crime Agency (SOCA) from the U.K. for their support throughout this project. This research was funded by the Research Councils UK Digital Economy Programme, Web Science Doctoral Training Centre, EP/G036926/1.

\section{REFERENCES}

[1] R. Albert, H. Jeong, and A. Barabasi, "Error and attack tolerance of complex networks," Nature, vol. 406, no. 6794, pp. 378-82, 27-Jul-2000.

[2] M. Arita, "Scale-Freeness and Biological Networks ," Journal of Biochemistry, vol. 138 , no. 1, pp. 1-4.

[3] E. Aumayr, J. Chan, and C. Hayes, "Reconstruction of Threaded Conversations in Online Discussion Forums," International AAAI Conference on Weblogs and Social Media; Fifth International AAAI Conference on Weblogs and Social Media, 2011.

[4] A.-L. Barabási and R. Albert, "Emergence of Scaling in Random Networks ," Science, vol. 286, no. 5439, pp. 509-512, Oct. 1999.

[5] A. Broder et al., "Graph structure in the Web," Computer Networks, vol. 33, no. 1-6, pp. 309-320, 2000.

[6] A. Clauset, C. R. Shalizi, and M. E. J. Newman, "Power-Law Distributions in Empirical Data," SIAM Review, vol. 51, no. 4, p. 661, Jun. 2009.

[7] V. Colizza, A. Flammini, M. A. Serrano, and A. Vespignani, "Detecting rich-club ordering in complex networks," Nat Phys, vol. 2, no. 2, pp. 110-115, Feb. 2006.

[8] J. Franklin, V. Paxson, A. Perrig, and S. Savage, "An inquiry into the nature and causes of the wealth of internet miscreants," in Proceedings of the 14th ACM conference on Computer and communications security, 2007, pp. 375-388.
[9] M. Glenny, Darkmarket: Cyberthieves, Cybercops and You. London: The Bodley Head, 2011.

[10] C. Herley and D. Florencio, "Nobody Sells Gold for the Price of Silver: Dishonesty, Uncertainty and the Underground Economy," Economics of Information Security and Privacy, pp. 33-53, 2010.

[11] M. O. Jackson and B. W. Rogers, "Meeting Strangers and Friends of Friends: How Random Are Social Networks?," American Economic Review, vol. 97, no. 3. pp. 890-915, 2007.

[12] A. Java, X. Song, T. Finin, and B. Tseng, "Why we twitter: understanding microblogging usage and communities," in Proceedings of the 9th WebKDD and 1st SNA-KDD 2007 workshop on Web mining and social network analysis, 2007, pp. 56-65.

[13] V. E. Krebs, "Mapping Networks of Terrorist Cells," Connections, vol. 24, no. 3, pp. 43-52, 2001.

[14] J. Leskovec and E. Horvitz, "Planetary-scale views on a large instantmessaging network" Proceeding of the 17th international conference on World Wide Web - WWW '08, p. 915, 2008.

[15] J. S. McIllwain, "Organized crime: A social network approach," Crime, Law and Social Change, vol. 32, no. 4, pp. 301-323, 1999.

[16] C. J. Mielke and H. Chen, "Botnets, and the cybercriminal underground," in Intelligence and Security Informatics, 2008. ISI 2008. IEEE International Conference on, 2008, pp. 206-211.

[17] T. Moore, R. Clayton, and R. Anderson, "The Economics of Online Crime," Journal of Economic Perspectives, vol. 23, no. 3. pp. 3-20, 2009.

[18] C. Morselli, C. Giguere, and K. Petit, "The efficiency/security trade-off in criminal networks," Social Networks, vol. 29, no. 1, pp. 143-153, Jan. 2007.

[19] M. Motoyama, D. McCoy, K. Levchenko, S. Savage, and G. M. Voelker, "An Analysis of Underground Forums," in Proceedings of the ACM Internet Measurement Conference (IMC), 2011.

[20] M. E. J. Newman, “Assortative Mixing in Networks," Physical Review Letters, vol. 89, no. 20, p. 208701, Oct. 2002.

[21] M. Newman, "Mixing patterns in networks," Physical Review E, vol. 67, no. 2, p. 14, Feb. 2003.

[22] M. E. J. Newman, Networks: An Introduction. New York, NY, USA: Oxford University Press, 2010.

[23] D. M. Pennock, G. W. Flake, S. Lawrence, E. J. Glover, and C. L. Giles, "Winners don't take all: Characterizing the competition for links on the web.," Proceedings of the National Academy of Sciences of the United States of America, vol. 99, no. 8, pp. 5207-11, Apr. 2002.

[24] K. K. Peretti, "Data Breaches: What the underground world of 'carding' reveals," Santa Clara Computer and High Technology Journal, vol. 25, pp. 375-414, 2008.

[25] E. Ravasz and A.-L. Barabási, "Hierarchical organization in complex networks," Physical Review E, vol. 67, no. 2, p. 26112, Feb. 2003.

[26] S. Sun, Z. Liu, Z. Chen, and Z. Yuan, "Error and attack tolerance of evolving networks with local preferential attachment," Physica A: Statistical Mechanics and its Applications, vol. 373, no. 0, pp. 851-860, Jan. 2007.

[27] R. Thomas and J. Martin, "the underground economy: priceless," The USENIX Magazine, vol. 31, no. 6, pp. 7-16, 2006.

[28] A. Vázquez and Y. Moreno, "Resilience to damage of graphs with degree correlations," Physical Review E, vol. 67, no. 1, p. 4, Jan. 2003.

[29] K. Wasserman, S. and Faust, Social Network Analysis: Methods and Applications (Structural Analysis in the Social Sciences). Cambridge University Press, 1994.

[30] D. J. Watts and S. H. Strogatz, "Collective dynamics of 'small-world' networks," Nature, vol. 393, no. 6684, pp. 440-442, Jun. 1998.

[31] J. Xu and H. Chen, "Untangling Criminal Networks: A Case Study," in ISI 2003, 2003, vol. 2665, p. 958.

[32] J. Xu and H. Chen, "The topology of dark networks," Communications of the ACM, vol. 51, no. 10, pp. 58-65, Oct. 2008.

[33] M. Yip, "An Investigation into Chinese Cybercrime and the Applicability of Social Network Analysis," in ACM Web Science Conference 2011, 14-17 June 2011, Koblenz, Germany., 2011. 